\title{
Estudos de externalidades na área de saúde humana decorrentes de reservatórios hidrelétricos
}

\section{Human health externalities studies derived from hydropower plants reservoirs}

Vinícius Verna M. Ferreira

Doutor em Saneamento, Meio Ambiente e Recursos Hídricos pela Universidade Federal de Minas Gerais (UFMG). Tecnologista Sênior do Centro de

Desenvolvimento da Tecnologia Nuclear (CDTN)

\section{Carlos Barreira Martinez}

Doutor em Planejamento de Sistemas Energéticos pela Universidade Estadual de Campinas (Unicamp). Professor do Departamento de Engenharia Hidráulica e Recursos Hídricos da UFMG

\section{Bruno Rabelo Versiani}

Doutor em Mecanique Des Milieux Géophysiques et Environnemen pela Université de Grenoble, França. Professor do Departamento de Engenharia

Hidráulica e Recursos Hídricos da UFMG

\section{Rosana Angélica M. Santos}

Engenheira Ambiental pela Fundação Mineira de Educação e Cultura (Fumec)

\section{Resumo}

O objetivo deste trabalho foi apresentar um método para se estimarem os valores das externalidades na área de saúde associadas à operação de reservatórios hidrelétricos. O método foi aplicado nos municípios de Uberaba e Uberlândia, Minas Gerais, parcialmente alagados pelo reservatório de Miranda. Foram analisados o número de internações hospitalares na rede do Sistema Único de Saúde e o crescimento populacional dos municípios alagados. Havendo relação de causa e efeito entre o lago e os impactos à saúde, o custo da externalidade em 2001 - quando US\$1 equivalia a R\$2,36 - foi estimado em $R \$ 25$ mil, sendo a população associada a esse valor de 773.409 pessoas. A valoração econômica dos impactos mostrou que os mesmos são pouco expressivos.

Palavras-chave: doenças associadas à água; externalidades; reservatórios.

\section{Abstract}

The objective of this work was to present an estimation method of the values of the health externalities associated to the operation of hydro power plants reservoirs. The method was applied in the cities of Uberaba and Uberlândia, Minas Gerais, Brazil, partially flooded by Miranda reservoir. The number of hospital admittances in the Public Health System and the population growth in the flooded municipal districts were analyzed. If there is a cause-effect relation between the lake and the diseases, the costs of externalities in 2001 were estimated in $R \$ 25,000$ when US\$ 1 corresponded to $R \$ 2,36$; the population number linked to this value was 773.409. The economical value of the health impacts showed that they do not have much significance.

Keywords: water-related diseases; externalities; reservoirs. 


\section{Introdução}

Um possível impacto oriundo da construção de grandes reservatórios é o aumento das doenças associadas à água. As barragens podem induzir diversas enfermidades, pois, muitas vezes, criam habitats ideais para a proliferação de mosquitos, caracóis e outros animais que servem como vetores para a disseminação dos micro-organismos geradores dessas moléstias. É comum em regiões próximas aos reservatórios o aumento dos casos de febre amarela, dengue, malária, esquistossomose, entre outros.

Todavia, em vários casos, mesmo cientes dos danos à saúde associados à construção de usinas hidrelétricas, autoridades decidiram desconsiderar os impactos devido ao alto custo das medidas mitigadoras (GARRETT, 1995). Como exemplo, no Senegal, mesmo sabendo da importância dos estudos de impacto à saúde humana, as autoridades competentes não incluíram esse tópico no planejamento das represas Diama e Manantali. Como resultado dessa omissão, surgiram várias epidemias, dentre as quais, a malária.

No Brasil, tomando-se como exemplo o início das obras de Tucuruí em 24/11/1975, observa-se que, a partir dessa data, ocorreu um significativo crescimento nos casos de malária, sendo que seu ápice ocorreu no ano de 1984, justamente quando se iniciou a operação comercial da usina. A média anual do número de casos no período anterior à construção da barragem (1962 a 1975) é de 168. Após a entrada em operação, a média subiu para 3.670 casos/ano (REIS, 2001). O crescimento dos casos de malária levou, inclusive, as comunidades indígenas da região a protestarem energicamente junto às autoridades competentes (KOIFMAN, 2001).

O aumento das doenças associadas à água é considerado uma externalidade negativa causada pela geração hidrelétrica. Entende-se como externalidade negativa os impactos trazidos por uma tecnologia de geração, cujos custos não são incorporados ao preço do produto e, consequentemente, não são repassados aos consumidores, sendo arcados por uma terceira parte, ou pela sociedade como um todo. A monetarização dessas externalidades dá origem aos custos externos ou socioambientais.

As principais externalidades negativas consideradas na geração de energia elétrica, pelas várias fontes e tecnologias empregadas, são os danos ao meio ambiente, às edificações e, principalmente, à saúde da população sob influência do empreendimento, além das emissões de gases de efeito estufa. A intensidade dos danos depende das características ambientais do local da usina, densidade populacional e condições meteorológicas, medidas de segurança empregadas e tecnologias utilizadas para a redução das emissões, entre outros fatores. Entretanto, embora os custos das externalidades negativas sejam reconhecidos pelos tomadores de decisão, muitas vezes esses valores são desconsiderados nas decisões políticas (BERTEL; FRASER, 2002).

\section{Metodologia}

Exemplifica-se a metodologia proposta por meio da usina hidrelétrica (UHE) de Miranda. Essa UHE situa-se no Estado de Minas Gerais, na região do Triângulo Mineiro, e entrou em operação em 1998. Seu reservatório alaga quatro municípios: Indianópolis, Nova Ponte, Uberaba e Uberlândia. Seu volume é de 145,6 milhões de metros cúbicos e sua potência nominal, de 408 MW (CEMIG, 2008). Os dados georeferenciados da região alagada foram obtidos junto ao setor de geoprocessamento da Agência Nacional de Energia Elétrica (ANEEL). A Tabela 1 apresenta alguns desses dados referentes à UHE e aos municípios parcialmente alagados pela mesma.

Para a obtenção do número de internações em hospitais do Sistema Único de Saúde (SUS) nos municípios alagados pela represa, foi realizada uma pesquisa referente ao ano de 2001, de acordo com a classificação existente na Classificação Internacional de Doenças (CID-10). A pesquisa limita-se aos casos registrados no SUS que resultaram em internações hospitalares devido à dificuldade de obtenção daqueles que não resultaram em internações. Uma dificuldade adicional é que não existe convergência entre os dados da Secretaria de Estado da Saúde de Minas Gerais, do SUS e da Fundação Nacional de Saúde (Funasa) no que se refere ao número de casos e/ou internações. Dessa forma, optou-se pelos dados do SUS, já que estes são de obtenção mais fácil, pois estão disponíveis na internet.

A CID fornece códigos relativos à classificação de doenças, e a cada estado de saúde é atribuída uma categoria única à qual corresponde um código (as categorias podem incluir um conjunto de doenças

Tabela 1 - Dados do lago de Miranda

\begin{tabular}{lcccc}
$\begin{array}{l}\text { UHE Miranda: } \\
\text { municípios alagados }\end{array}$ & $\begin{array}{c}\text { Área dos municípios } \\
\left(\mathbf{k m}^{2}\right)\end{array}$ & $\begin{array}{c}\text { Área municipal alagada } \\
\left(\mathbf{k m}^{2}\right)\end{array}$ & $\begin{array}{c}\text { Relação área alagada/ } \\
\text { não alagada }(\%)\end{array}$ & $\begin{array}{c}\text { Área municipal não } \\
\text { alagada }\left(\mathbf{k m}^{2}\right)\end{array}$ \\
\hline Indianópolis & 833,87 & 21,04 & 2,58 & 812,82 \\
\hline Nova Ponte & $1.105,77$ & 7,27 & 0,66 & $1.098,49$ \\
\hline Uberaba & $4.512,14$ & 3,14 & 0,07 & $4.508,99$ \\
\hline Uberlândia & $4.115,82$ & 20,89 & 0,51 & $4.094,92$ \\
\hline Total & $10.567,60$ & 52,36 & 0,49 & $10.515,23$ \\
\hline
\end{tabular}

UHE: usina hidrelétrica.

Fonte: ANEEL (2004). 
semelhantes, agrupadas de modo a facilitar as estatísticas correspondentes). A CID é publicada pela Organização Mundial de Saúde (OMS), sendo revisada periodicamente e usada globalmente para estatísticas de morbidade e mortalidade, sistemas de reembolso e decisões automáticas de suporte em Medicina, entre outras aplicações.

Como fonte para os dados hospitalares, foi utilizado o portal do Departamento de Informática do Sistema Único de Saúde (Datasus) (2008), que possui os dados discriminados em nível municipal desde 1992. O sistema em foco corresponde a hospitais públicos (federais, estaduais e municipais), universitários (públicos ou privados), filantrópicos e contratados à iniciativa privada. Todavia, cabe ressaltar que há algumas limitações nos dados associados as Autorizações de Internações Hospitalares (AIH) existentes na base do Datasus. Algumas dessas são o perfil dos pacientes - não incluindo aqueles em piores condições de vida -, a ausência de padronização e treinamento para classificação das doenças ou lesões que motivaram a internação e o problema de a unidade de análise ser a internação e não o indivíduo, de modo que as doenças que requeiram mais de uma internação podem aparecer em frequências mais altas do que a sua real prevalência na população (PEREIRA, 1999).

Como os dados utilizados restringem-se ao SUS, para contabilizar as internações ocorridas em clínicas e hospitais particulares, foi aplicada uma metodologia na qual se considera a relação entre a taxa de mortalidade dada pelo Sistema de Informações de Mortalidade (SIM $=6,74)$ e a taxa de mortalidade no âmbito do Sistema de Informações Hospitalares (SIH/SUS = 1,95). Também foi contabilizada a porcentagem de óbitos não informada ao sistema de saúde (16,55\%), que é obtida da relação entre os óbitos informados, presentes no SIM, e aqueles obtidos por meio dos censos demográficos do Instituto Brasileiro de Geografia e Estatística (IBGE). Dessa forma, obtém-se um fator de ajuste igual a 4,14, conforme observado na Equação 1.

$\mathrm{FA}=[(6,74 / 1,95) \times 100 /(100-16,55)]=4,14$

Onde:

FA: fator de ajuste.

Esse valor foi adotado devido à ausência de dados similares referentes à morbidade. Ressalta-se aqui que esses valores referem-se ao ano de
2001, escolhido para exemplificar a metodologia aplicada para a estimação das externalidades. Para que esse método seja utilizado em outros anos, é necessária a obtenção dos valores associados aos mesmos.

\section{Resultados}

A Tabela 2 apresenta os dados do número de internações devido a enfermidades associadas à água nos municípios de Uberaba e Uberlândia, alagados pela represa de Miranda, segundo o Datasus. Para ilustrar a metodologia aplicada, foram escolhidas cinco enfermidades: leishmaniose tegumentar, leishmaniose visceral, cólera, esquistossomose e leptospirose. Em Indianópolis e Nova Ponte, não foram registradas internações no período em estudo, sendo então possível que os habitantes dessas localidades procurem atendimento em municípios próximos que ofereçam uma melhor infraestrutura na área de saúde, tais como Araguari, Uberlândia e Uberaba.

A partir da CID-10, a leishmaniose passou a ser subdividida em cutânea, cutâneo-mucosa, visceral e não especificada. Neste trabalho, o termo leishmaniose tegumentar representa as duas primeiras formas citadas, e a leptospirose engloba as três subdivisões existentes na CID-10: icterohemorrágica, não especificada e outras formas de leptospirose.

O custo total das externalidades negativas na área de saúde foi calculado de acordo com a metodologia abaixo apresentada (REIS, 2001):

- $\quad$ custo total $=$ gastos com internações + gastos ambulatoriais + custo dos dias perdidos;

- $\quad$ custo dos dias perdidos = (número de internações com idade acima de 14 anos x média de permanência) x renda média mensal/30;

- $\quad$ renda média em Minas Gerais: R\$ 430,00 (valor de 2001)

A idade de 15 anos foi considerada como sendo a base para o cálculo dos dias perdidos, de acordo com os dados do IBGE, os quais mostram que a população economicamente ativa a partir dessa faixa etária é maior do que a inativa. Em relação à idade, cabe ressaltar aqui que o decreto $\mathrm{n}^{\circ} 4.134$, de 15 de fevereiro de 2002, promulga a convenção $n^{\circ} 138$ e a recomendação $n^{\circ} 146$ da Organização Internacional do Trabalho (OIT) sobre idade mínima de admissão ao emprego ou trabalho. $\mathrm{O}$ artigo estabelece que essa idade mínima

Tabela 2 - Número de internações - dados do Datasus

\begin{tabular}{lcc} 
Enfermidade/ano & Uberaba (2001) & Uberlândia (2001) \\
\hline Leishmaniose visceral & 1 & 2 \\
\hline Leishmaniose tegumentar & 3 & 7 \\
\hline Esquistossomose & 4 & 2 \\
\hline Cólera & 1 & 2 \\
\hline Leptospirose & 1 & 2 \\
\hline
\end{tabular}


é de 16 anos. Todavia, as planilhas do IBGE incluem as idades de 15 e 16 anos dentro do mesmo grupo. Assim, para compatibilizar essas duas informações, adotou-se neste trabalho 15 anos como a idade mínima para cálculos da população economicamente ativa, para se contabilizarem os dias perdidos quando da valoração das externalidades negativas.

Para obtenção dos gastos ambulatoriais por doença, utilizou-se a relação entre os gastos médios no SUS por internação e por atendimento ambulatorial; dessa forma, o valor presente na coluna gastos com internações (Tabela 3) é dividido por 98,04, conforme pode ser visto na Equação 2.

Gastos ambulatoriais por doença $=$

$\frac{266,69 \text { ( gasto médio por internação) }}{2,72 \text { (gasto médio por atendimento ambulatorial) }}=98,04$

Os resultados finais obtidos estimam o valor da externalidade associado às enfermidades em estudo (Tabela 3).
Para que os valores apresentados na Tabela 3 não fiquem restritos ao sistema previdenciário, os mesmos foram multiplicados pelo fator de ajuste igual a 4,14 , e os resultados obtidos são apresentados na Tabela 4

Conforme pode ser observado na Tabela 5, algumas enfermidades associadas à água já existiam na região antes mesmo da construção da UHE de Miranda. Essa tabela apresenta o somatório das internações nos municípios de Uberaba e Uberlândia, ocorridas nos seis anos anteriores e nos seis primeiros anos de operação da usina. Assim, no caso da comprovação da existência de uma externalidade negativa, esta será representada pelo percentual superior ao número médio de internações após a construção do reservatório, dentro da metodologia proposta. Analogamente, nos casos em que houve uma redução do número de internações, é possível que tal fato seja oriundo dos benefícios decorrentes da implantação da represa e, portanto, seja uma externalidade positiva da mesma. Todavia, a avaliação dessas possíveis externalidades positivas foge ao objetivo deste trabalho.

Tabela 3 - Valoração das externalidades: usina hidrelétrica de Miranda (ano base 2001)

\begin{tabular}{lccccc}
\multicolumn{1}{c}{ Enfermidade } & $\begin{array}{c}\text { Número de } \\
\text { internacónes } \\
\text { no SUS* }\end{array}$ & $\begin{array}{c}\text { Média de } \\
\text { permanência } \\
\text { (dias) }\end{array}$ & $\begin{array}{c}\text { Gastos com } \\
\text { internações } \\
\text { (reais) }\end{array}$ & $\begin{array}{c}\text { Gastos } \\
\text { ambulatoriais } \\
\text { (reais) }\end{array}$ & $\begin{array}{c}\text { Dias } \\
\text { perdidos } \\
\text { (reais) }\end{array}$ \\
$\begin{array}{l}\text { Leishmaniose } \\
\text { tegumentar }\end{array}$ & 10 & 30,6 & $4.534,60$ & 46,24 & $4.386,00$ \\
(reais) \\
total
\end{tabular}

*Pessoas com idade superior a 14 anos.

Tabela 4 - Valor das externalidades de saúde: usina hidrelétrica de Miranda (ano base 2001)

\begin{tabular}{|c|c|c|c|}
\hline Enfermidade & Valor (reais) & Número de casos & Custo por caso (reais) \\
\hline Leishmaniose tegumentar & $37.122,75$ & 10 & $3.712,27$ \\
\hline Leishmaniose visceral & $10.773,86$ & 3 & $3.591,28$ \\
\hline Esquistossomose & $22.572,74$ & 6 & $3.762,12$ \\
\hline Cólera & 895,98 & 1 & 895,98 \\
\hline Leptospirose & $5.369,14$ & 2 & $1.789,71$ \\
\hline
\end{tabular}

Tabela 5 - Somatório do número de internações

\begin{tabular}{lcc}
\hline Enfermidade/Ano & 1992 a 1997 & 1998 a 2003 \\
\hline Leishmaniose visceral + tegumentar* & 30 \\
\hline Esquistossomose & 35 & 22 \\
\hline Cólera & 1 & 19 \\
\hline Leptospirose & 13 \\
\hline
\end{tabular}

* Doenças agrupadas, visto que a CID-9 não diferenciava os tipos. 
Analisando-se os dados apresentados na Tabela 5, as enfermidades que apresentam um crescimento no número de internações hospitalares, em relação ao período anterior à construção da UHE, são a cólera e a leishmaniose. Deste modo, caso o aumento dessas internações após a construção da UHE de Miranda se deva exclusivamente à presença da barragem, o valor total da externalidade na área de saúde para o ano de 2001, de acordo com a metodologia aplicada, é estimado em $\mathrm{R} \$ 25.744,79$, e o valor endêmico total das mesmas doenças, em $\mathrm{R} \$ 23.047,79$, conforme apresentado na Tabela 6. Esses números foram obtidos multiplicando-se o valor dos custos das externalidades de saúde, presentes na Tabela 4, pela fração correspondente associada aos valores "endêmicos" e "não endêmicos".

Como exemplo, no caso da leishmaniose, sendo 1,68 o número das internações em 2001 (valor expresso por 100 mil habitantes) e 0,8 o número médio de internações antes da construção da UHE, o número de internações associado a essa possível externalidade é de $0,88(1,68-0,8)$, o que equivale a $52,4 \%$ do total das internações e dos custos.

\section{Tratamento estatístico}

Efetuando-se uma análise comparativa entre o número de internações devido às doenças associadas à água, nas cidades alagadas pela UHE de Miranda e em todo o Estado de Minas Gerais, obtêm-se os resultados apresentados na Tabela 7. A análise foi feita em dois períodos de seis anos: o primeiro entre 1992 e 1997 e o segundo, entre 1998 e 2003. $\mathrm{Na}$ análise estudaram-se intervalos iguais antes e depois da entrada em operação da UHE de Miranda. Os limites inferior/superior dos intervalos de confiança foram obtidos por meio do teste $t$ de Student, sendo que a distribuição dos dados foi considerada normal. Para cada enfermidade, foram calculados a média do número de internações e seu desvio padrão, para dois períodos diferentes de seis anos (HAAN, 1977).

Optou-se por trabalhar com um intervalo de confiança de 95\%; ou seja, a probabilidade de o resultado desejado (número médio de internações) não estar contido no intervalo especificado é de apenas 5\%.

De acordo com a Tabela 7, o aumento das internações devido à cólera na região alagada pela UHE de Miranda foi 37,5\% inferior

Tabela 6 - Valor da externalidade relativa às doenças associadas à água para a usina hidrelétrica (UHE) de Miranda (ano base 2001)

\begin{tabular}{llc} 
Valoração de externalidades/enfermidade & Leishmaniose & Cólera \\
\hline Número médio de internações antes da construção da UHE (fonte: Datasus) & 0,8 & 0,03 \\
\hline Internações em 2001 - SUS (a) & 1,68 & 0,13 \\
\hline Custo total (reais) & $47.896,61$ & 895,98 \\
\hline Valor endêmico (reais) & $22.856,09$ & 191,70 \\
\hline Valor da externalidade (reais) & $25.040,52$ & 704,27 \\
\hline
\end{tabular}

(a): Valores expressos por 100.000 habitantes, sendo a população total de 773.409 pessoas.

Tabela 7 - Avaliação comparativa usina hidrelétrica (UHE) de Miranda/Estado de Minas Gerais

\begin{tabular}{|c|c|c|}
\hline \multirow{2}{*}{ Enfermidade/Região } & \multicolumn{2}{|c|}{ Internações* (Intervalo de confiança de 95\%) } \\
\hline & 1992-1997 & $1998-2003$ \\
\hline \multicolumn{3}{|l|}{ Cólera } \\
\hline Minas Gerais & $0,08(0,0-0,16)$ & $0,19(0,0-0,40)$ \\
\hline UHE de Miranda & $0,03(0,0-0,08)$ & $0,06(0,0-0,14)$ \\
\hline \multicolumn{3}{|l|}{ Leishmaniose } \\
\hline Minas Gerais & $2,06(1,82-2,30)$ & $1,88(1,63-2,13)$ \\
\hline UHE de Miranda & $0,80(0,56-1,04)$ & $1,46(1,34-1,59)$ \\
\hline \multicolumn{3}{|l|}{ Esquistossomose } \\
\hline Minas Gerais & $3,84(2,56-5,12)$ & $1,32(1,21-1,42)$ \\
\hline UHE de Miranda & $0,93(0,58-1,28)$ & $0,49(0,19-0,79)$ \\
\hline \multicolumn{3}{|l|}{ Leptospirose } \\
\hline Minas Gerais & $0,65(0,54-0,76)$ & $0,47(0,39-0,56)$ \\
\hline UHE de Miranda & $0,51(0,22-0,80)$ & $0,28(0,11-0,45)$ \\
\hline
\end{tabular}

*Valores expressos por 100.000 habitantes. 
Tabela 8 - Teste estatístico unilateral direito

\begin{tabular}{|lcc|}
\hline Enfermidade & Leishmaniose & Cólera \\
\hline Número médio de internações antes da construção da UHE de Miranda & 0,8 & 0,03 \\
\hline Número médio de internações após a construção da UHE de Miranda & 1,46 & 0,06 \\
\hline Desvio padrão do número de internações após a construção da UHE & 0,17 & 0,11 \\
\hline Número de anos estudados & 6 & 9,44 \\
\hline Resultado do teste unilateral direito (Equação 2) & 2,02 & 0,69 \\
\hline Valor tabelado & Rejeitada & Aceita \\
\hline Hipótese & & 6 \\
\hline
\end{tabular}

UHE: usina hidrelétrica.

ao aumento do índice do Estado. No que se refere à leishmaniose, observa-se que o número de internações no Estado é maior do que na região circunvizinha à UHE de Miranda. Entretanto, os dados atestam que em todo o Estado o número de internações na rede do SUS relativas a essa enfermidade decresceu $8,74 \%$, enquanto que nos municípios alagados esse mesmo número cresceu 82,5\%.

Para verificar se existe uma concordância estatística entre a teoria e os fatos, aplicou-se um teste unilateral direito aos resultados obtidos. O teste foi efetuado visando verificar estatisticamente se realmente ocorreu um aumento no número de internações associadas a essas doenças. Caso isso tenha ocorrido, estas podem vir a ser classificadas como possíveis externalidades negativas. A hipótese que se desejou testar é se os valores médios do número de internações obtidos, antes e após a construção da usina, são estatisticamente iguais (UNIVERSIDADE TÉCNICA DE LISBOA, 2004). Para tanto, aplicou-se a Equação 3:

$$
\mathrm{P}=\frac{\mathrm{X} 1-\mathrm{X} 0}{\frac{\mathrm{DP}}{\sqrt{\mathrm{N}}}}
$$

Onde:

P: probabilidade;

Xl: incidência média após a construção da UHE de Miranda;

X0: incidência média antes da construção da UHE de Miranda;

DP: desvio padrão do número de internações após a construção da UHE de Miranda;

$\mathrm{N}$ : número de anos estudados.

Cada um dos valores obtidos (um para cada enfermidade) foi comparado com os dados tabelados da distribuição de $t$ de Student. Nesta análise, também foi considerado um intervalo de confiança de 95\%. Caso o valor encontrado seja maior que o tabelado, a hipótese de as médias serem iguais é rejeitada (AYYUB; MCCUEN, 1997).

Analisando-se os resultados obtidos, verifica-se que, no caso da leishmaniose, a hipótese de as médias das incidências antes e após a construção da UHE de Miranda serem iguais foi rejeitada. Todavia, para a cólera, a hipótese foi aceita (Tabela 8).

Dessa forma, de acordo com o teste, as médias das internações devido à cólera antes e após a construção da UHE de Miranda não sofreram alteração e, portanto, essa enfermidade deve ser excluída da lista de possíveis externalidades negativas. Ressalta-se aqui que estudos locais constataram que a água da represa também é utilizada sem tratamento para consumo doméstico (BORGES, 2006), o que aumenta o risco da incidência de cólera na região. Entretanto, mesmo com a exclusão da cólera, o valor da externalidade negativa anteriormente calculado diminui muito pouco, permanecendo seu total na casa dos R 25 mil. Ressalta-se aqui que não é possível aplicar o teste estatístico para as outras enfermidades em estudo, visto que nos outros casos, conforme visto na Tabela 6 , ocorreu uma diminuição no número de internações após a construção da UHE de Miranda. Contudo, vale lembrar que só os dados estatísticos não estabelecem uma associação causa-efeito. Para que a associação seja causal, deve-se levar em consideração vários critérios já consagrados, tais como consistência, intensidade, especificidade, relação temporal, gradiente e coerência.

\section{Valoração das externalidades}

Dentro da metodologia proposta, para se analisar a significância dos resultados obtidos, comparou-se primeiramente o valor da externalidade com o custo de geração da UHE de Miranda. De acordo com dados obtidos junto à gerência de planejamento de mercado da Companhia Energética de Minas Gerais (CEMIG), a geração de energia de Miranda no ano de 2001 foi de 1.090.056 MWh, e a tarifa média das usinas hidrelétricas nesse mesmo ano foi de $\mathrm{R} \$ 41,00$. O custo da externalidade obtido foi de 0,023 R\$/MWh, correspondendo a 0,056\% do valor da tarifa de energia elétrica.

De acordo com dados obtidos no site do IBGE, obteve-se o Produto Interno Bruto (PIB), ano base 2001, dos municípios de Uberaba e Uberlândia, cujo somatório tem o valor de R\$ 8.295.708,00. O PIB representa a soma, em valores monetários, de todos os bens e serviços 
finais produzidos em uma determinada região durante um período determinado de tempo. De acordo com a metodologia aplicada, o valor total da externalidade negativa obtida corresponde a cerca de 0,3\% do valor do PIB somado dos dois municípios citados.

Segundo a base do Datasus, no ano de 2001 houve 4.670 internações hospitalares nos dois municípios em estudo, para uma população total de 773.409 pessoas. Dessa forma, a fração de internações correspondente a essa possível externalidade negativa é praticamente nula. Também segundo o Datasus, os gastos totais referentes às internações hospitalares nesses mesmos municípios são da ordem de R\$ 3.879.678,06, sendo, então, o valor da externalidade negativa calculada é inferior a 1\% desse montante.

De acordo com o Datasus, nenhuma dessas internações resultou em óbito. Caso isso houvesse ocorrido, o cálculo da valoração das externalidades seria sobremaneira dificultado. Ressalta-se aqui que algumas possibilidades precisam ser mais bem estudadas para que conclusões efetivas sobre o real motivo do crescimento do número de internações devido à leishmaniose sejam obtidas, antes de se considerar a barragem de Miranda como a causa desse fato. Outros pesquisadores têm estudado a questão dessa enfermidade no município de Uberlândia há algum tempo, e esta parece ser endêmica na região, visto que anualmente são registrados casos da doença. Fatores que ratificam essa afirmação são a grande quantidade de flebotomíneos capturados anualmente no local e o significativo número de casos da doença registrados também entre cães (MAYWALD et al., 1996; MACHADO; MAYWALD; LEMOS, 2006). Além disso, em locais próximos às barragens das hidrelétricas de Capim Branco I e II, situadas entre os municípios de Araguari e Uberlândia, esse problema também se mostra relevante, existindo grande quantidade de mosquitos transmissores da leishmaniose no local (LEMOS, 2007).

O estudo das externalidades constitui uma linha de pesquisa que, apesar de pouco explorada, pode fornecer subsídios importantes para estudos comparativos entre as diversas possibilidades existentes para a geração de energia elétrica. Outras externalidades associadas às usinas hidrelétricas não foram incorporadas a esta análise, mas também vêm sendo estudadas, como a perda de produção agropecuária devido à área inundada (FERREIRA; MARTINEZ; VERSIANI, 2008) e as emissões de gases de efeito estufa em reservatórios (FERREIRA MARTINEZ; VERSIANI, 2006).

\section{Conclusões}

Apresentou-se neste trabalho uma estimativa do cálculo de externalidades associadas à saúde humana decorrentes da construção de reservatórios hidrelétricos. Verificou-se que o número de internações devido à leishmaniose aumentou no período posterior à construção do reservatório da UHE de Miranda; todavia, existem indícios de essa doença ser endêmica na região, pois anualmente causa internações, mesmo antes da construção da represa.

De qualquer forma, considerando-se hipoteticamente que o aumento da incidência dessas moléstias seja integralmente oriundo dos impactos gerados pelo reservatório, observa-se que seu valor é bastante pequeno quando comparado à tarifa de energia elétrica e ao PIB dos municípios de Uberaba e Uberlândia, parcialmente inundados pelo lago de Miranda. Do mesmo modo, quando comparado ao número total de internações hospitalares, independentemente de sua causa, e aos gastos totais associados às mesmas, constata-se que o impacto dessa possível externalidade negativa é pouco expressivo.

Cabe ressaltar que, segundo a OMS, apesar de a cólera ser uma doença de notificação compulsória, apenas 2\% dos casos na América do Sul são efetivamente relatados (LEWINSOHN, 2003), sendo o problema comum a outras enfermidades. Desse modo, os valores encontrados neste trabalho podem ser conservativos.

\section{Referências}

AGÊNCIA NACIONAL DE ENERGIA ELÉTRICA (ANEEL). Disponível em www.aneel.gov.br. Acesso em: 18 fev. 2008.

AYYUB, B.M.; MCCUEN, R.H. Probability, Statistics, and Reliability for Engineers. New York: CRC Press, 1997.

BERTEL, E.; FRASER, P. Energy Policy and Externalities. NEA Updates, NEA News, v. 20, n. 1, p. 14-17, 2002.

BORGES, A.A.S. Diagnóstico ambiental e proposta de monitoramento da área do lago de Miranda pertencente ao município de Indianópolis - MG. Dissertação (Mestrado em Geografia). Universidade Federal de Uberlândia, 2006.

COMPANHIA ENERGÉTICA DE MINAS GERAIS (CEMIG). 2008. Disponível em: www.cemig.com.br. Acesso em: 18 fev. 2008.
DEPARTAMENTO DE INFORMÁTICA DO SISTEMA ÚNICO DE SAÚDE (DATASUS). 2008. Disponível em: www.datasus.gov.br. Acesso em: 18 set. 2008.

FERREIRA, V.V.M.; MARTINEZ, C.B.; VERSIANI, B.R. Metodologia para estimação de externalidades agropecuárias decorrentes da construção de reservatórios. Engenharia Sanitária e Ambiental, v. 13, n. 1, p. 29-37, 2008.

Avaliação das emissões de gases de efeito estufa derivadas de reservatórios de usinas hidrelétricas em Minas Gerais. Revista Brasileira de Recursos Hídricos, v. 11, n. 3, p. 113-122, 2006.

GARRETT, L. A próxima peste: novas doenças num mundo em desequilíbrio. Rio de Janeiro: Nova Fronteira, 1995. 
HAAN, C.T. Statistical Methods in Hydrology. Ames, lowa: The lowa State University Press, 1977.

KOIFMAN, S. Geração e transmissão da energia elétrica: impacto sobre os povos indígenas no Brasil. Cadernos de Saúde Pública, v.17, n.2, p. 413-423, 2001.

LEMOS, J.C. Fauna flebotomínea na Bacia do Rio Araguari, antes, durante e após a construção da barragem da Usina Hidrelétrica Capim Branco I, Uberlândia, Minas Gerais, Brasil. Tese (Doutorado em Geografia). Universidade Federal de Uberlândia, 2007.

LEWINSOHN, R. Três Epidemias: lições do passado. Campinas: Editora da Unicamp, 2003.

MACHADO, M. I.; MAYWALD, P. G.; LEMOS, J. C. Vigilância epidemiológica da leishmaniose tegumentar americana (LTA) nas áreas de influência e entorno da Usina Hidrelétrica de Miranda, MG. VIII Simpósio Ítalo-
Brasileiro de Engenharia Sanitária e Ambiental. CD Rom. Fortaleza - CE. 2006.

MAYWALD, P.G. et al. Leishmaniose tegumentar, visceral e doença de Chagas caninas em municípios do Triângulo Mineiro e Alto Paranaíba, Minas Gerais, Brasil. Cadernos de Saúde Pública, v. 12, n. 3, p. 321 328, 1996.

PEREIRA, M.G. Epidemiologia: teoria e prática. Rio de Janeiro: Guanabara Koogan, 1999.

REIS, M.M. Custos ambientais associados à geração elétrica: hidrelétricas x termelétricas a gás natural. Dissertação (Mestrado em Planejamento Energético). Universidade Federal do Rio de Janeiro, 2001.

UNIVERSIDADE TÉCNICA DE LISBOA. 2004. Disponível em: http:// www.fmv.utl.pt/departamentos/dsa/biomatematica/files/aulas/ aulas015e016_20e21Abr2004.pdf. Acesso em: 06 ago. 2004. 